Alfred Maussner ${ }^{\mathrm{a}}$ and Julius Spatz ${ }^{\mathrm{b}}$

\title{
Determinants of Business Cycles in Small Scale Macroeconomic Models: The German Case
}

\begin{abstract}
August $2005^{\text {cd }}$
Abstract

We identify measures of shocks to total factor productivity and preferences from two real business cycle models and subject them to Granger causality tests to see whether they can be considered exogenous to other plausible sources of the German business cycle. For West German data from 1960.i to 1989.iv we conclude that our measures of shocks are indeed exogenous. This contrasts with similar studies for other countries that question the exogeneity of either productivity or preference shocks. For the period 70.i to 01.iv we find that M3 Granger causes all of our shock measures. We attribute this to the breaks in our time series associated with the German reunification in 1990 and the European Monetary Union in 1999.
\end{abstract}

Keywords Real Business Cycles, Solow Residual, Granger Causality

\section{JEL Classification E32, O47}

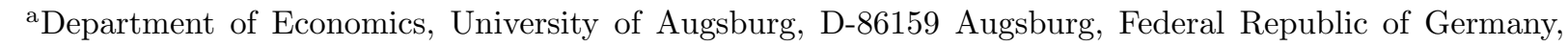
Email alfred.maussner@wiwi.uni-augsburg.de,www.wiwi/uni-augsburg.de/vwl/maussner/

${ }^{\mathrm{b}}$ Deutsche Gesellschaft für Technische Zusammenarbeit (GTZ) GmbH, Postfach 5180, D-65726 Eschborn, Federal Republic of Germany, Email julius.spatz@gtz.de

${ }^{c}$ Earlier versions of this paper circulate as University of Augsburg Economics Discussion Paper No. 213 and Kiel Institute for World Economics Working Paper No. 1158, respectively.

${ }^{\mathrm{d}}$ We gratefully acknowledge the comments of two anonymous referees. All remaining errors are ours. 


\section{Appendix}

Figure A.1: Time Path of Hours 1960-1989
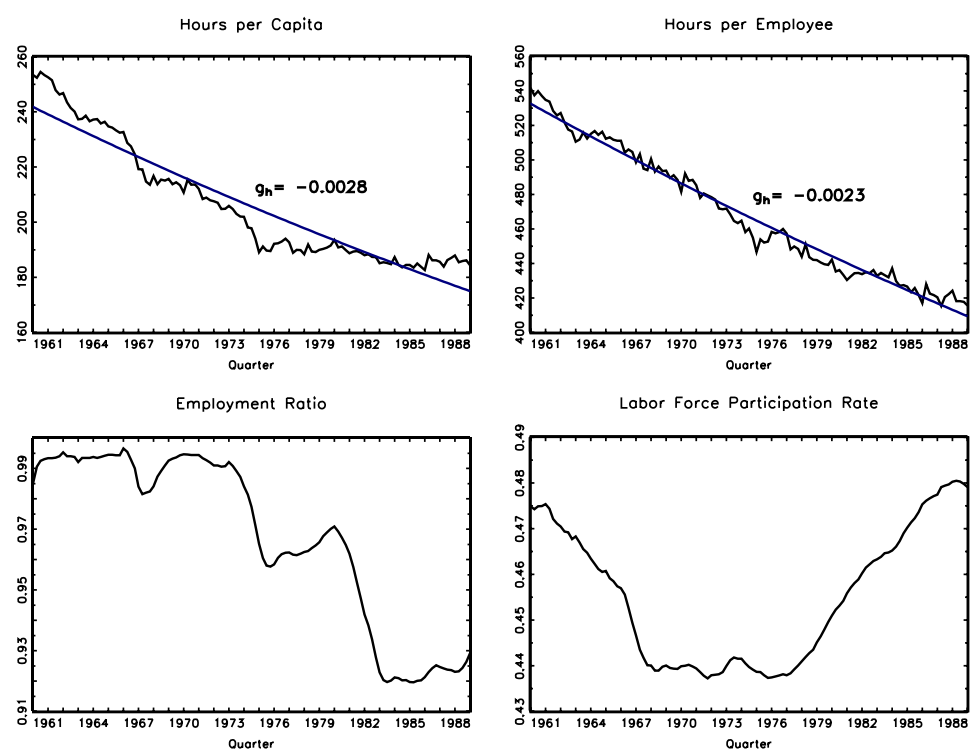

Figure A.2: Time Path of Hours 1976-1989
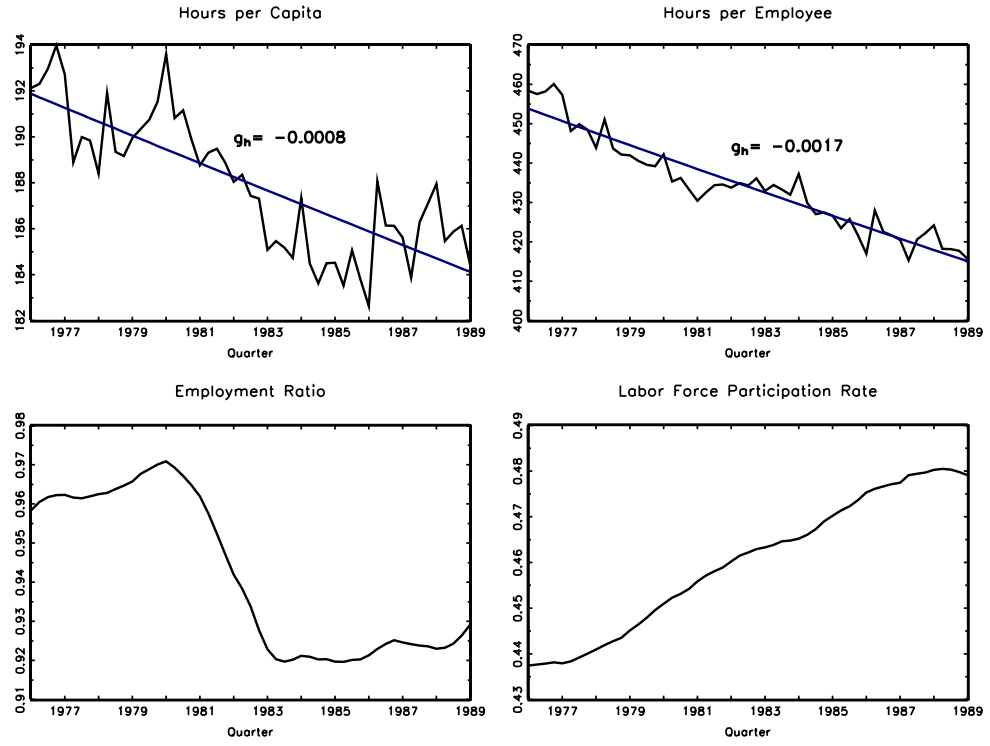
Figure A.3: Time Path of Hours 1971-2001
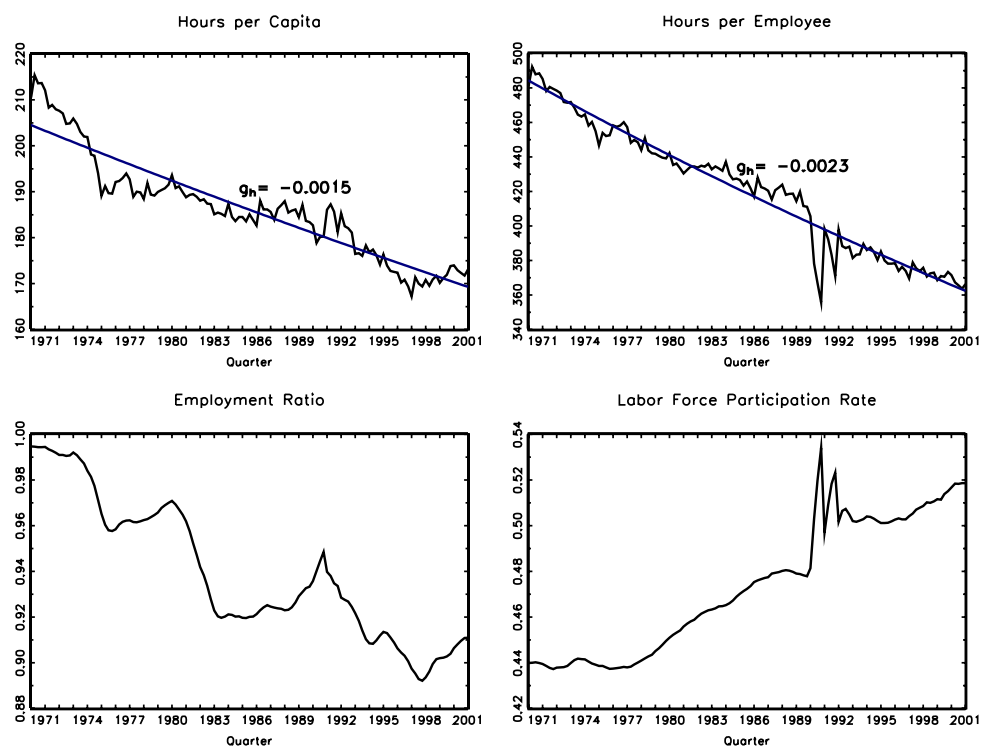
Figure A.4: Time Paths of Test Variables
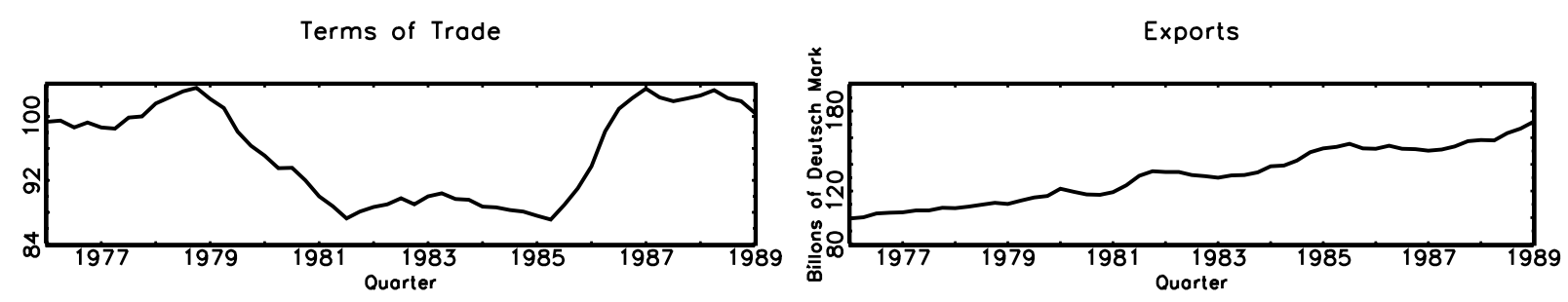

M1
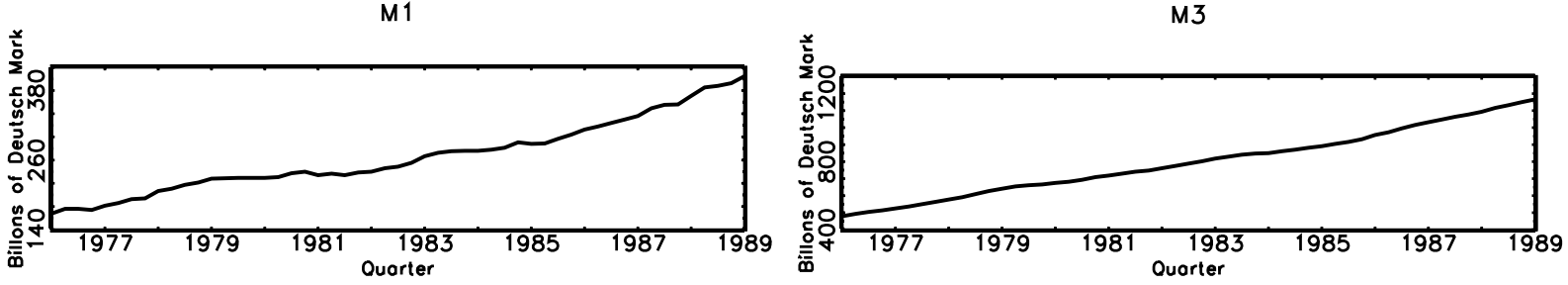

Short Term Interest Rate

Long Term Interest Rote
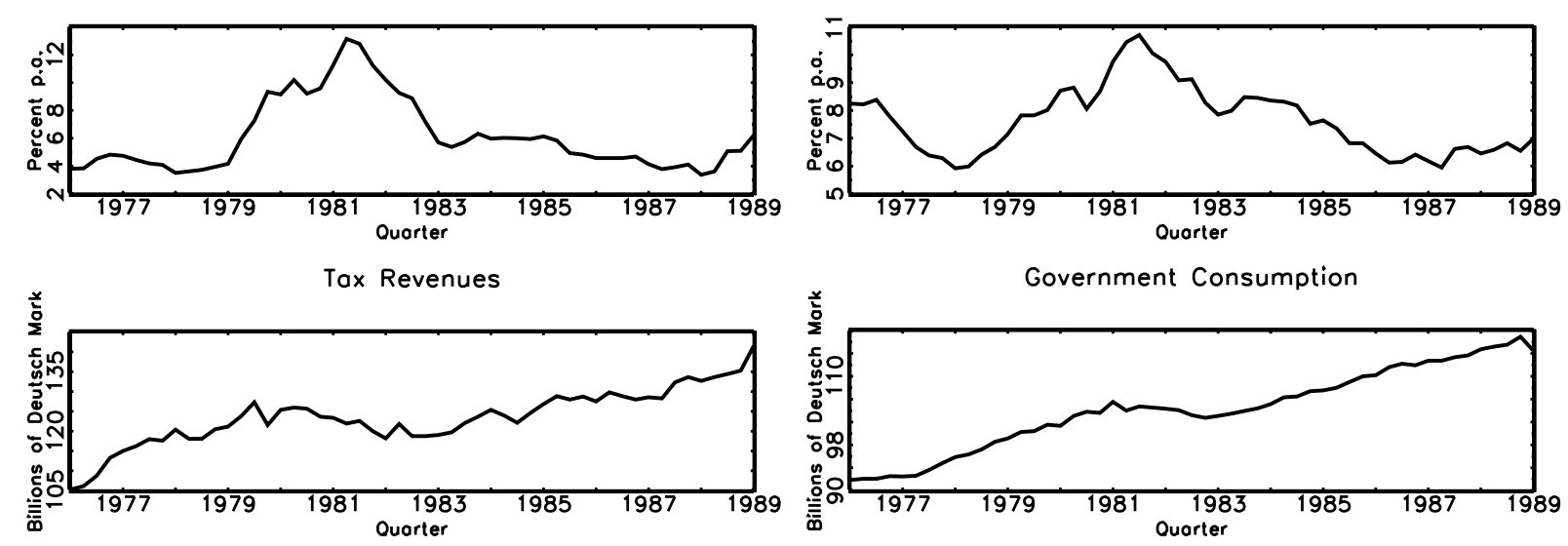
Table A.1:

Second Moments of Simulated Time Series

\begin{tabular}{llllllllll}
\hline \hline & \multicolumn{3}{c}{ Empirical Time Series } & \multicolumn{3}{c}{ Model One } & \multicolumn{3}{c}{ Model Two } \\
Variable & $s_{x}$ & $r_{x}$ & $r_{x y}$ & $s_{x}$ & $r_{x}$ & $r_{x y}$ & $s_{x}$ & $r_{x}$ & $r_{x y}$ \\
\hline Output & 1.12 & 0.80 & 1.00 & 1.52 & 0.36 & 1.00 & 2.03 & 0.48 & 1.00 \\
Consumption & 1.25 & 0.85 & 0.82 & 1.01 & 0.22 & 0.88 & 1.02 & 0.23 & 0.88 \\
Investment & 2.71 & 0.80 & 0.80 & 4.97 & 0.48 & 0.97 & 8.18 & 0.57 & 0.98 \\
Hours & 0.77 & 0.31 & 0.37 & 1.70 & 0.64 & 0.81 & 1.92 & 0.60 & 0.88 \\
Real Wage & 1.24 & 0.91 & 0.47 & 0.99 & 0.17 & 0.14 & 1.08 & 0.11 & 0.37 \\
Real Interest Rate & 0.37 & 0.43 & -0.11 & 1.65 & 0.64 & 0.72 & 1.52 & 0.63 & 0.05 \\
\hline
\end{tabular}

Notes: $s_{x}:=$ Standard deviation of HP-filtered time series of variable $x, r_{x}:=$ first-order autocorrelation of variable $x, r_{x y}:=$ cross-correlation of variable $x$ with output.

The moments of the simulated series are averages over 500 simulations with 55 observations each.

The simulations use the parameters displayed in column two of Table ??, $\eta=1.5$ and the estimated standard deviation of the growth rate of the productivity shock, $\sigma_{\epsilon}=0.01006\left(\sigma_{\epsilon}=0.01027\right.$ for model two) as well as the estimated parameters from $\mathrm{AR}(1)$-processes fitted to the logarithmic deviation of the preference shock and the oil-price shock from its respective means, $\rho_{\theta}=0.93, \sigma_{\theta}=0.00996\left(\rho_{\theta}=0.86\right.$, $\sigma_{\theta}=0.00901$ for model two), $\rho_{p}=0.95, \sigma_{p}=0.14497$. 
Table A.2:

Unit Root Tests 76.i-89.iv

\begin{tabular}{lccccc}
\hline \hline Variable & & Levels & \multicolumn{2}{c}{ First Differences } \\
& ADF & PP & KPSS & ADF & PP \\
& & & & & \\
Productivity Shock (1) & -1.449 & -1.817 & 0.118 & $-2.958^{* *}$ & $-8.680^{* * *}$ \\
Productivity Shock (2) & $-3.676^{* *}$ & -2.522 & 0.105 & $-3.886^{* * *}$ & $-7.376^{* * *}$ \\
Preference Shock (1) & -2.012 & -2.601 & $0.613^{* *}$ & $-10.092^{* * *}$ & $-9.906^{* * *}$ \\
Preference Shock (2) & -1.992 & -2.445 & 0.268 & $-3.318^{* *}$ & $-9.648^{* * *}$ \\
Government Expenditures & -2.602 & -1.518 & 0.097 & $-3.530^{* *}$ & $-7.560^{* * *}$ \\
Taxes & -2.322 & -2.569 & 0.109 & $-7.769^{* * *}$ & $-7.757^{* * *}$ \\
M1 & -1.793 & -2.149 & 0.109 & $-7.082^{* * *}$ & $-7.124^{* * *}$ \\
M3 & -2.424 & -2.826 & $0.147^{* *}$ & $-2.806^{* *}$ & $-3.939^{* * *}$ \\
Short-Term Interest Rate & -1.934 & -1.847 & 0.136 & $-4.423^{* * *}$ & $-4.496^{* * *}$ \\
Long-Term Interest Rate & -1.322 & -1.821 & 0.201 & $-5.140^{* * *}$ & $-5.154^{* * *}$ \\
Exports & $-3.255^{* * *}$ & -2.535 & 0.062 & $-5.977^{* * *}$ & $-5.847^{* * *}$ \\
Terms of Trade & -0.776 & -1.356 & $0.173^{* *}$ & -1.963 & $-3.373^{* *}$ \\
Oil Price & -1.812 & -1.394 & 0.190 & $-4.752^{* * *}$ & $-4.525^{* * *}$ \\
\hline
\end{tabular}

\section{Notes:}

ADF: The augmented Dickey-Fuller t-statistic. The estimated model is

$$
\Delta x_{t}=m+b t+r x_{t-1}+\sum_{i=1}^{q} a_{i} \Delta x_{t-i}+\epsilon_{t}
$$

and the true process is

$$
\Delta x_{t}=\mu+\sum_{i=1}^{q} \alpha_{i} \Delta x_{t-i}+\epsilon_{t},
$$

where $x_{t}$ refers to $\log$ of the variable in column 1 , except in the case of interest rates. $q$ in equation (i) was chosen to minimize the Schwarz information criterium over $p=1,2, \ldots, \bar{p}, \bar{p}=\left[12(T / 100)^{1 / 4}\right]$, where $[z]$ denotes the integer part of $z$ and $T$ denotes the sample size, respectively (see Hayashi, 2000, p. 594 on this choice of $\bar{p}$ ). The test statistic is the t-statistic of the estimated $r$. Critical values are from MacKinnon (1991).

PP: Phillips-Peron $Z_{t}$ statistic with lag truncation parameter equal to 7 . The estimated model and the true process are as in (i) and (ii). The critical values are the same as those of the ADF-t statistic.

KPSS: Kwiatkowski-Phillips-Schmidt-Shin statistic of the null of stationarity. The bandwidth parameter was set to 7, critical values are taken from Kwiatkowski et al. (1992), Table 1.

The ADF-t and PP tests for the first differences of the variables in column 1 estimate the model (i) without the time trend $b t$ and assume (ii) without the drift term $\mu$.

$*, * *$, or $* * *$ denote rejection of the null at the $10 \%, 5 \%$, or $1 \%$ level. 
Table A.3:

Unit Root Tests 60.i-89.iv

\begin{tabular}{lrrrrc}
\hline \hline Variable & & Levels & \multicolumn{2}{c}{ First Differences } \\
& ADF & PP & KPSS & ADF & PP \\
& & & & \\
Productivity Shock (1) & -1.011 & -1.230 & $0.381^{* * *}$ & $-3.730^{* * *}$ & $-10.838^{* * *}$ \\
Productivity Shock (2) & 0.016 & -0.427 & $0.381^{* * *}$ & $-5.823^{* * *}$ & $-10.258^{* * *}$ \\
Preference Shock (1) & -1.911 & -1.973 & $1.459^{* * *}$ & $-4.342^{* * *}$ & $-13.081^{* * *}$ \\
Preference Shock (2) & -2.803 & -2.775 & $1.106^{* * *}$ & $-4.950^{* * *}$ & $-12.763^{* * *}$ \\
Government Expenditures & -0.682 & -0.682 & $0.374^{* * *}$ & $-9.745^{* * *}$ & $-10.149^{* * *}$ \\
Taxes & -2.742 & -2.699 & $0.347^{* * *}$ & $-13.117^{* * *}$ & $-13.049^{* * *}$ \\
M1 & -2.103 & -2.232 & $0.196^{* *}$ & $-4.566^{* * *}$ & $-13.242^{* * *}$ \\
M3 & 0.463 & 0.297 & $0.388^{* * *}$ & $-1.831^{* * *}$ & $-4.924^{* * *}$ \\
Short-Term Interest Rate & $-4.335^{* * *}$ & -2.911 & $0.211^{* * *}$ & $-4.986^{* * *}$ & $-7.646^{* * *}$ \\
Long-Term Interest Rate & -2.506 & -2.261 & $0.326^{* * *}$ & $-7.311^{* * *}$ & $-7.226^{* * *}$ \\
Exports & -0.510 & -1.396 & $0.372^{* * *}$ & $-2.404^{* *}$ & $-12.089^{* * *}$ \\
Terms of Trade & -1.914 & -1.869 & $0.202^{* *}$ & $-3.354^{* * *}$ & $-8.047^{* * *}$ \\
Oil Price & -1.874 & -1.572 & $1.243^{* * *}$ & $-7.729^{* * *}$ & $-7.475^{* * *}$ \\
\hline
\end{tabular}

Notes: See Table A.2 
Table A.4:

Unit Root Tests 70.i-01.iv

\begin{tabular}{llll}
\hline \hline Variable & t-Statistic & Variable & t-Statistic \\
& & & \\
Productivity Shock (1) & -2.552 & Productivity Shock (M2) & -2.522 \\
Preference Shock (1) & -2.131 & Preference Shock (M2) & -1.999 \\
Government Expenditures & -2.847 & Taxes & -3.352 \\
M1 & -2.785 & M3 & -2.456 \\
Short-Term Interest Rate & $-3.47{ }^{*}$ & Long Term Interest Rate & -2.988 \\
Exports & -2.151 & Terms of Trade & -2.589 \\
Oil Price & -2.650 & & \\
\hline
\end{tabular}

\section{Notes:}

a) The estimated model is

$$
\tilde{x}_{t}=r \tilde{x}_{t-1}+\sum_{i=1}^{q} a_{i} \Delta \tilde{x}_{t-i}+\epsilon_{t}
$$

where $\tilde{x}_{t}$ is the OLS-residual from the regression

$$
x_{t}=c+\delta d_{t}+\beta t+\eta_{t} .
$$

The dummy variable $d_{t}$ is zero for quarters $70 . \mathrm{i}$ through 90 .iv and one for the remaining quarters. Columns 2 and 3 report the t-statistic of $H_{0}: r=1$. For our sample size and choice of the breakpoint the $10 \%$, $5 \%$, and $1 \%$ critical values are $-3.46,-3.76$, and -4.32 , respectively (see, Perron (1989), Table IV.B).

b) $*, * *$, or $* * *$ denote rejection of the null at the $10 \%, 5 \%$, or $1 \%$ level. 
Table A.5:

Cointegration Tests 60.i-89.iv

\begin{tabular}{|c|c|c|c|c|c|c|c|c|}
\hline \multirow[t]{4}{*}{ Variable } & \multicolumn{8}{|c|}{ Model One Shock Measures } \\
\hline & \multicolumn{4}{|c|}{ Productivity } & \multicolumn{4}{|c|}{ Preferences } \\
\hline & \multicolumn{2}{|c|}{ AIC } & \multicolumn{2}{|c|}{ HQ } & \multicolumn{2}{|c|}{$\mathrm{AIC}$} & \multicolumn{2}{|c|}{ HQ } \\
\hline & $q$ & $r_{1}, r_{2}$ & $q$ & $r_{1}, r_{2}$ & $q$ & $r_{1}, r_{2}$ & $q$ & $r_{1}, r_{2}$ \\
\hline Government Expenditures & 7 & 1,1 & 5 & 1,1 & 5 & 2,0 & 1 & 1,1 \\
\hline Taxes & 6 & 1,1 & 1 & 2,2 & 4 & 0,0 & 1 & 0,0 \\
\hline M1 & 6 & 0,0 & 6 & 0,0 & 7 & 0,0 & 6 & 0,0 \\
\hline M3 & 6 & 0,0 & 6 & 0,0 & 8 & 2,2 & 6 & 2,0 \\
\hline Short-Term Interest Rate & 6 & 0,0 & 5 & 2,0 & 5 & 1,1 & 5 & 1,1 \\
\hline Long-Term Interest Rate & 6 & 0,0 & 5 & 0,0 & 4 & 0,0 & 2 & 1,0 \\
\hline Exports & 6 & 0,0 & 6 & 0,0 & 6 & 1,0 & 5 & 0,0 \\
\hline Terms of Trade & 6 & 0,0 & 6 & 0,0 & 6 & 0,0 & 6 & 0,0 \\
\hline Oil Price & 5 & 1,1 & 2 & 1,1 & 4 & 0,0 & 2 & 0,0 \\
\hline \multirow[t]{4}{*}{ Variable } & \multicolumn{8}{|c|}{ Model Two Shock Measures } \\
\hline & \multicolumn{4}{|c|}{ Productivity } & \multicolumn{4}{|c|}{ Preferences } \\
\hline & \multicolumn{2}{|r|}{$\mathrm{AIC}$} & \multicolumn{2}{|c|}{ HQ } & \multicolumn{2}{|c|}{$\mathrm{AIC}$} & \multicolumn{2}{|c|}{ HQ } \\
\hline & $q$ & $r_{1}, r_{2}$ & $q$ & $r_{1}, r_{2}$ & $q$ & $r_{1}, r_{2}$ & $q$ & $r_{1}, r_{2}$ \\
\hline Government Expenditures & 8 & 2,2 & 1 & 2,2 & 5 & 2,0 & 1 & 1,0 \\
\hline Taxes & 5 & 1,1 & 1 & 2,2 & 4 & 0,0 & 4 & 0,0 \\
\hline M1 & 6 & 0,0 & 6 & 0,0 & 6 & 1,1 & 6 & 1,1 \\
\hline M3 & 6 & 0,0 & 6 & 0,0 & 6 & 2,0 & 6 & 2,0 \\
\hline Short-Term Interest Rate & 5 & 2,0 & 5 & 2,0 & 5 & 1,1 & 5 & 1,1 \\
\hline Long-Term Interest Rate & 6 & 2,0 & 2 & 0,0 & 4 & 0,0 & 4 & 0,0 \\
\hline Exports & 5 & 0,0 & 5 & 0,0 & 6 & 1,0 & 5 & 0,0 \\
\hline Terms of Trade & 6 & 0,0 & 6 & 0,0 & 6 & 0,0 & 6 & 0,0 \\
\hline
\end{tabular}

Notes:

AIC and HQ refer to the Akaike and the Hannan-Quinn information criterium, respectively. $q$ is the number of lags in the estimated model selected by AIC or HQ. $r_{1}\left(r_{2}\right)$ indicates the cointegrating rank according to the Johansen trace (maximum Eigenvalue) test. 
Table A.6:

Cointegration Tests 76.i-89.iv

\begin{tabular}{|c|c|c|c|c|c|c|c|c|}
\hline \multirow[t]{4}{*}{ Variable } & \multicolumn{8}{|c|}{ Model One Shock Measures } \\
\hline & \multicolumn{4}{|c|}{ Productivity } & \multicolumn{4}{|c|}{ Preferences } \\
\hline & \multicolumn{2}{|r|}{$\mathrm{AIC}$} & \multicolumn{2}{|c|}{ HQ } & \multicolumn{2}{|c|}{$\mathrm{AIC}$} & \multicolumn{2}{|c|}{ HQ } \\
\hline & $q$ & $r_{1}, r_{2}$ & $q$ & $r_{1}, r_{2}$ & $q$ & $r_{1}, r_{2}$ & $q$ & $r_{1}, r_{2}$ \\
\hline Government Expenditures & 8 & 0,0 & 1 & 0,0 & 5 & 0,0 & 5 & 0,0 \\
\hline Taxes & 8 & 0,0 & 5 & 0,0 & 4 & 0,0 & 1 & 0,0 \\
\hline M1 & 8 & 1,1 & 1 & 0,0 & 4 & 0,0 & 1 & 0,0 \\
\hline M3 & 8 & 1,1 & 5 & 0,0 & 5 & 0,0 & 5 & 0,0 \\
\hline Short-Term Interest Rate & 5 & 0,0 & 5 & 0,0 & 2 & 0,0 & 2 & 0,0 \\
\hline Long-Term Interest Rate & 5 & 0,0 & 1 & 0,0 & 3 & 0,0 & 1 & 0,0 \\
\hline Exports & 5 & 0,0 & 5 & 0,0 & 8 & 0,0 & 2 & 0,0 \\
\hline Terms of Trade & 6 & 0,0 & 6 & 0,0 & 6 & 0,0 & 5 & 0,0 \\
\hline Oil Price & 5 & 0,0 & 2 & 0,0 & 4 & 0,0 & 2 & 0,0 \\
\hline \multirow[t]{4}{*}{ Variable } & \multicolumn{8}{|c|}{ Model Two Shock Measures } \\
\hline & \multicolumn{4}{|c|}{ Productivity } & \multicolumn{4}{|c|}{ Preferences } \\
\hline & \multicolumn{2}{|c|}{$\mathrm{AIC}$} & \multicolumn{2}{|c|}{ HQ } & \multicolumn{2}{|c|}{$\mathrm{AIC}$} & \multicolumn{2}{|c|}{ HQ } \\
\hline & $q$ & $r_{1}, r_{2}$ & $q$ & $r_{1}, r_{2}$ & $q$ & $r_{1}, r_{2}$ & $q$ & $r_{1}, r_{2}$ \\
\hline Government Expenditures & 1 & 0,0 & 1 & 0,0 & 8 & 1,1 & 5 & 0,0 \\
\hline Taxes & 5 & 0,0 & 1 & 0,0 & 4 & 0,0 & 1 & 0,0 \\
\hline M1 & 1 & 0,1 & 1 & 0,1 & 4 & 0,0 & 1 & 0,0 \\
\hline M3 & 8 & 1,1 & 8 & 1,1 & 5 & 0,0 & 5 & 0,0 \\
\hline Short-Term Interest Rate & 5 & 0,0 & 5 & 0,0 & 2 & 0,0 & 2 & 0,0 \\
\hline Long-Term Interest Rate & 1 & 0,0 & 1 & 0,0 & 3 & 2,0 & 1 & 0,0 \\
\hline Exports & 5 & 0,0 & 1 & 0,0 & 8 & 0,0 & 2 & 0,0 \\
\hline Terms of Trade & 6 & 0,0 & 6 & 0,0 & 6 & 0,0 & 5 & 1,1 \\
\hline
\end{tabular}

Notes: See Table A.5. 
Table A.7:

Cointegration Tests 70.i-01.iv

\begin{tabular}{|c|c|c|c|c|c|c|c|c|}
\hline \multirow[t]{4}{*}{ Variable } & \multicolumn{8}{|c|}{ Model One Shock Measures } \\
\hline & \multicolumn{4}{|c|}{ Productivity } & \multicolumn{4}{|c|}{ Preferences } \\
\hline & \multicolumn{2}{|c|}{$\mathrm{AIC}$} & \multicolumn{2}{|c|}{ HQ } & \multicolumn{2}{|c|}{$\mathrm{AIC}$} & \multicolumn{2}{|c|}{ HQ } \\
\hline & $q$ & $r_{1}, r_{2}$ & $q$ & $r_{1}, r_{2}$ & $q$ & $r_{1}, r_{2}$ & $q$ & $r_{1}, r_{2}$ \\
\hline Government Expenditures & 7 & 1,1 & 4 & 1,1 & 5 & 1,1 & 1 & 1,1 \\
\hline Taxes & 2 & 1,1 & 2 & 1,1 & 8 & 1,1 & 1 & 1,1 \\
\hline M1 & 6 & 1,1 & 3 & 1,1 & 4 & 0,0 & 4 & 0,0 \\
\hline M3 & 6 & 1,1 & 3 & 2,2 & 4 & 0,0 & 4 & 0,0 \\
\hline Short-Term Interest Rate & 2 & 1,1 & 2 & 1,1 & 5 & 1,1 & 2 & 1,1 \\
\hline Long-Term Interest Rate & 2 & 1,1 & 2 & 1,1 & 4 & 1,0 & 2 & 1,1 \\
\hline Exports & 5 & 0,0 & 5 & 0,0 & 5 & 0,0 & 5 & 0,0 \\
\hline Terms of Trade & 6 & 1,1 & 2 & 0,0 & 6 & 0,0 & 6 & 0,0 \\
\hline Oil Price & 6 & 0,0 & 2 & 0,0 & 6 & 0,0 & 5 & 0,0 \\
\hline
\end{tabular}

Variable

Model Two Shock Measures

\begin{tabular}{ccccccc}
\multicolumn{2}{c}{ Productivity } & \multicolumn{3}{c}{ Preferences } \\
AIC & HQ & & AIC & \multicolumn{1}{c}{ HQ } \\
$r_{1}, r_{2}$ & $q$ & $r_{1}, r_{2}$ & $q$ & $r_{1}, r_{2}$ & $q$ & $r_{1}, r_{2}$
\end{tabular}

$\begin{array}{lllllllll}\text { Government Expenditures } & 5 & 1,1 & 4 & 1,1 & 7 & 1,1 & 2 & 2,0\end{array}$

$\begin{array}{lllllllll}\text { Taxes } & 6 & 1,1 & 2 & 1,1 & 8 & 2,2 & 1 & 2,0\end{array}$

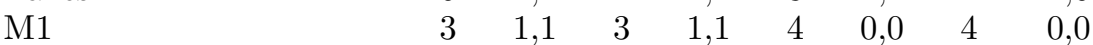

M3 $\begin{array}{llllllll}3 & 1,1 & 3 & 1,1 & 4 & 1,1 & 4 & 1,1\end{array}$

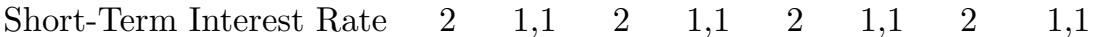

$\begin{array}{lllllllll}\text { Long-Term Interest Rate } & 2 & 1,1 & 2 & 1,1 & 4 & 1,1 & 2 & 1,1\end{array}$

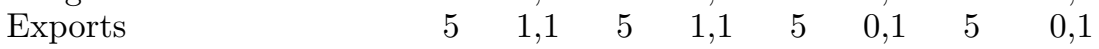

$\begin{array}{lllllllll}\text { Terms of Trade } & 7 & 1,1 & 2 & 0,0 & 6 & 0,0 & 2 & 0,0\end{array}$

Notes: See also Table A.5. Since all tests were performed with a dummy variable that accounts for the break in 1991, the critical values for the Johansen tests are at best indicative of the existence of cointegration. 
Table A.8:

Multivariate Granger Causality Tests 70.i-01.iv: Error Correction, r=1

\begin{tabular}{lccccc}
\hline \hline Shock & $q$ & $\mathrm{M} 1 \rightarrow$ & $\rightarrow \mathrm{M} 1$ & $\mathrm{M} 3 \rightarrow$ & $\rightarrow \mathrm{M} 4$ \\
& & & & & \\
Productivity shock (1) & 3.00 & 0.41 & 0.14 & 0.00 & 0.23 \\
Productivity shock (2) & 3.00 & 0.12 & 0.19 & 0.00 & 0.42 \\
Preference shock (1) & 4.00 & 0.59 & 0.88 & 0.00 & 0.98 \\
Preference shock (2) & 4.00 & 0.17 & 0.82 & 0.00 & 0.96 \\
\hline
\end{tabular}

Notes: $q$ denotes the lag length of the estimated VAR in levels. In all four tests both the AIC and the HQ criterium selected the same lag length. The column labeled M1 $\rightarrow$ $(\mathrm{M} 3 \rightarrow)$ displays the marginal level of significance for the null that M1 (M3) Granger causes the shock in column 1 . The column labeled $\rightarrow \mathrm{M} 1(\rightarrow \mathrm{M} 3)$ displays the marginal level of significance for the null that the shock from column1 1 Granger causes M1 (M3). 